\title{
DEPRESIÓN EN PACIENTTES CON TUBERCULOSIS EN UNA REGIÓN DEL PERÚ: UN ESTUDIO TRANSVERSAL
}

\author{
DEPRESSION AMONG PATIENTS WITH TUBERCULOSIS \\ IN A REGION OF PERU: A CROSS-SECTIONAL STUDY
}

\author{
CÉSAR ANTONIO BONILLA ASALDE \\ Universidad Norbert Wiener
}

ISABEL CRISTINA RIVERA LOZADA

Universidad del Cauca, Colombia

ORIANA RIVERA LOZADA

Universidad Norbert Wiener

\section{RESUMEN}

La tuberculosis, además de producir deterioro clínico, causa problemas sociales y mentales, con mayor frecuencia la depresión, que afecta negativamente al proceso del tratamiento. El presente estudio tuvo como objetivo determinar los niveles de depresión en pacientes con tuberculosis en una región de alta carga de la enfermedad en el Perú. Es un estudio transversal analítico en 110 pacientes con tuberculosis. Se recolectaron datos sociodemográficos y clínicos mediante una encuesta; se aplicó el cuestionario de salud del paciente (PHQ-9) para diagnosticar depresión. El porcentaje de depresión entre los pacientes con tuberculosis fue del 54,5\%; fue encontrada una relación estadísticamente significativa entre la depresión y género, estado civil, escolaridad, ingreso económico, tipo de tuberculosis y estatus del tratamiento. El estudio permitió concluir que la depresión es común en los pacientes con tuberculosis, lo que sugiere que el personal de salud tenga en cuenta esta situación al evaluar a los pacientes con tuberculosis; la depresión no diagnosticada puede amenazar la eficacia de la estrategia DOTS.

Palabras clave: tuberculosis, depresión, tratamiento, salud mental.

\section{ABSTRACT}

Tuberculosis, besides producing clinical deterioration, causes social and mental problems, more frequently depression which affects the treatment process negatively. The objective of this study was to determine the level of depression in patients with tuberculosis in a region with high rates of this disease in Peru. It is an analytical cross-sectional study on 110 patients with tuberculosis. Data collection included sociodemographic and clinical data obtained through a survey; we applied the PHQ-9 questionnaire to diagnose depression. The percentage of depression among the patients with tuberculosis was $54.5 \%$. We found a statistically significant relationship between depression and gender, civil status, schooling, economic 
income, type of tuberculosis and treatment status. The study allowed us to conclude that depression is common among patients with tuberculosis, which suggests that health personnel should take this situation into account to evaluate patients with tuberculosis; undiagnosed depression can threaten the efficacy of the DOTS strategy.

Key words: tuberculosis, depression, treatment, mental health.

\section{INTRODUCCIÓN}

La tuberculosis (TB) es una enfermedad infecciosa causada por Mycobacterium tuberculosis ${ }^{1,2}$ y se encuentra entre las principales causas de morbilidad y mortalidad en el mundo ${ }^{1,3}$. Según la OMS, en 2018 se estimaron -aproximadamente- 10 millones de casos y 1.2 millones de muertes ${ }^{4,5}$.

En el Perú, para ese periodo, la morbilidad fue de 32642 casos de TB en todas sus formas, de los cuales el $60 \%$ fueron registrados en Lima y Callao, con una tasa de mortalidad de 4,2 por 100000 habitantes $^{6}$.

La tuberculosis no solo produce deterioro clínico; además, causa problemas sociales y mentales ${ }^{7,8}$. Los trastornos mentales representan el $13 \%$ de toda la carga de la enfermedad; la depresión es la cuarta causa en todo el mundo y se prevé que para 2020 sea la segunda causa principal de carga de enfermedad mental ${ }^{9,10}$.

La depresión se caracteriza por tristeza, perdida de interés o placer, baja autoestima, sueño o apetito alterado, sentimientos de cansancio y falta de concentración ${ }^{11}$. Cuando se presenta en TB no permite un buen resultado del tratamiento, ocasionando discapacidad y mala calidad de vida en quien la padece ${ }^{12}$.

La evidencia muestra que la prevalencia de depresión en pacientes con TB es más alta que en la población general, por lo que esta relación debe ser abordada como un problema importante de salud pública, que va a influir en el deterioro no solo clínico, sino también social de los afectados por esta comorbilidad ${ }^{13-19}$.

Un estudio de Sudáfrica mostró que la prevalencia de depresión era del 32,9 \% ${ }^{13}$. Para el caso de Nigeria, los niveles de depresión oscilaron entre el $27,7 \%$ y el $45,5 \%{ }^{15,16}$, y en Etiopía, se informó que el $54 \%$ de los pacientes con TB tenían depresión ${ }^{12}$. La edad avanzada, el nivel de educación, el apoyo social, el sexo femenino, el estigma de TB percibido y la duración de la enfermedad fueron algunos factores identificados como asociados con la depresión ${ }^{12-16}$. Dos estudios de Pakistán estimaron que la prevalencia de depresión en pacientes con TB-MDR fue de 69,6\% y $42,8 \%{ }^{20.21}$.

En el Perú, un estudio del año 2013 evidenció un nivel de depresión de $37 \%$ en pacientes con $\mathrm{TB}$, asociado a resultados negativos del tratamiento ${ }^{22}$.

Las personas afectadas por tuberculosis desarrollan depresión debido a factores como la larga duración del tratamiento, la estigmatización que sufre el paciente debido a la enfermedad y la falta de apoyo familiar. En el Perú, la carga de salud mental no se conoce bien $^{23}$ y aunque hay algunos estudios disponibles con respecto a 
la depresión, la presente investigación tuvo como objetivo determinar los niveles de depresión en pacientes con tuberculosis en una región de alta carga de la enfermedad en el país.

\section{MATERIAL Y MÉTODOS}

Se llevó a cabo un estudio transversal analítico en pacientes que recibían tratamiento para tuberculosis en establecimientos de salud de la región del Callao, y que consultaron en el Hospital Daniel Alcides Carrión durante el periodo febrero-agosto de 2018. Como criterios de exclusión fueron considerados los diagnósticos previos con depresión y la no firma del consentimiento informado.

Se incluyeron en el estudio 110 pacientes, mediante un muestreo no probabilístico que recolectó datos sociodemográficos y clínicos a través de una encuesta. Se aplicó el cuestionario de salud del paciente (PHQ-9) para diagnosticar depresión ${ }^{24}$.

PHQ-9 es un cuestionario autoinformado de nueve ítems y de buena aceptación y alta confiabilidad por detectar trastornos depresivos ${ }^{24-27}$; la puntuación va de 0 a 27 y tiene puntos de corte que permiten establecer rangos de depresión leve, moderado y grave.

Una vez recogidos los datos por los investigadores, fueron ingresados a una base de datos en Excel y luego exportados al paquete estadístico IBM SPSS versión 25 . Se calcularon las medidas de frecuencia y de tendencia central de acuerdo a la naturaleza de las variables y se utilizó la prueba de chi cuadrado para evaluar la asociación entre variables categóricas; un $\mathrm{p}<0,05$ se consideró significativo.
Este trabajo fue evaluado y aprobado por el comité de ética del Hospital Daniel Alcides Carrión de la región del Callao.

\section{RESULTADOS}

En el estudio participaron 110 pacientes que se encontraban recibiendo tratamiento para tuberculosis en establecimientos de salud de la región Callao y que consultaron en el Hospital Daniel Alcides Carrión de la región Callao en el Perú.

En el análisis descriptivo se evidenció que el 61,8\% de los encuestados pertenecía al género masculino, la mediana de edad fue 33 años $+/-2$, el 46,4\% tenía como grado de escolaridad la secundaria y el 69,1\% de la población tenía un ingreso económico familiar no mayor a 930 soles (véase la Tabla 1).

En la Tabla 2 se observa que el 54,5\% de la población del estudio presentaba algún nivel de depresión. El 32,7 \% de los pacientes presentaron depresión leve y un 7,3 $\%$ presentaba una depresión severa-moderada de acuerdo a lo evaluado por la escala de depresión PHQ-9.

Se encontró asociación estadísticamente significativa $(\mathrm{p}<0,05)$ entre las características de la población como género, estado civil, escolaridad, ingreso económico familiar, tipo de tuberculosis, estatus del tratamiento y la depresión (véase la Tabla 3). 
TABLA 1

Características sociodemográficas de la población

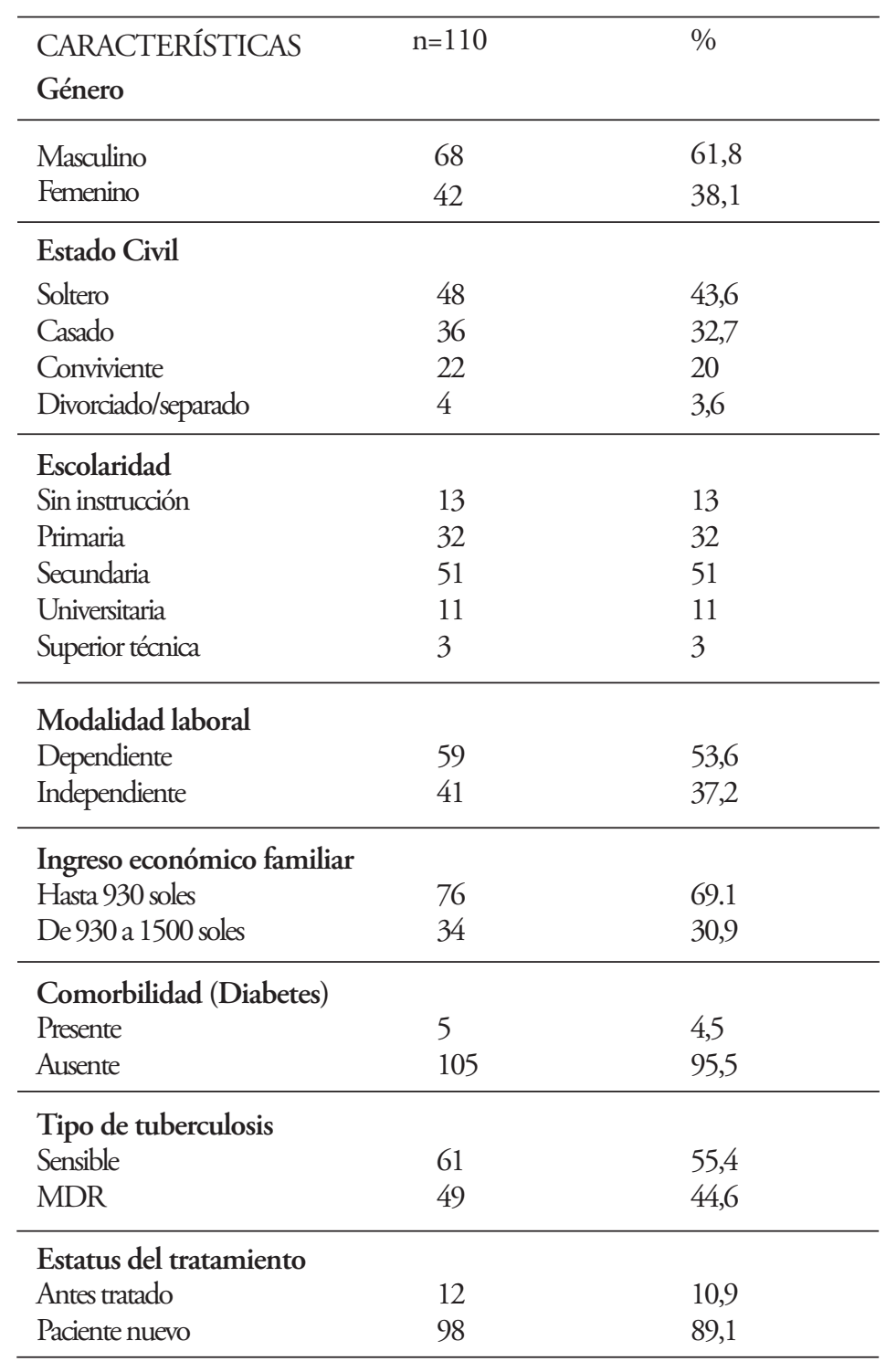


César Antonio Bonilla Asalde, Isabel Cristina Rivera Lozada, Oriana Rivera Lozada

TABLA 2

Depresión y severidad de acuerdo a la escala PHQ-9 en pacientes con tuberculosis

\begin{tabular}{llll}
\hline Depresión y severidad & Puntaje & N & Porcentaje (\%) \\
\hline No/Mínima & $0-4$ & 50 & 45,4 \\
Leve & $5-9$ & 36 & 32,7 \\
moderada & $10-14$ & 16 & 14,5 \\
Severa moderada & $15-19$ & 8 & 7,3 \\
\hline
\end{tabular}

TABLA 3

Características de la población y depresión al tratamiento

\begin{tabular}{|c|c|c|c|c|c|}
\hline \multirow[b]{2}{*}{ CARACTERÍSTICAS } & \multicolumn{2}{|c|}{$\begin{array}{c}\text { Depresión } \\
\mathrm{n}(\%)\end{array}$} & \multicolumn{2}{|c|}{ Total } & \multirow[t]{2}{*}{ Valor p. } \\
\hline & Presente & Ausente & $\mathbf{n}$ & $\%$ & \\
\hline \multicolumn{6}{|l|}{ Género } \\
\hline Masculino & $9(13,2)$ & $59(86,8)$ & 68 & $(100)$ & 0,02 \\
\hline Femenino & $13(31,0)$ & $29(69,0)$ & 42 & $(100)$ & \\
\hline \multicolumn{6}{|l|}{ Estado civil } \\
\hline Soltero & $19(39,6)$ & $29(60,4)$ & 48 & $(100)$ & 0,01 \\
\hline Divorciado/separado & & & & & \\
\hline Casado/Conviviente & $11(17,7)$ & $51(82,3)$ & 62 & $(100)$ & \\
\hline \multicolumn{6}{|l|}{ Escolaridad } \\
\hline Sin instrucción & $8(61,5)$ & $5(38,5)$ & 13 & $(100)$ & 0,00 \\
\hline Con instrucción & $21(21,6)$ & $76(78,4)$ & 97 & $(100)$ & \\
\hline \multicolumn{6}{|l|}{ Ingreso económico familiar } \\
\hline Hasta 930 soles & $25(32,9)$ & $4(80,0)$ & 76 & & 0,04 \\
\hline De 930 a 1500 soles & $4(13,3)$ & $80(76,2)$ & 34 & & \\
\hline \multicolumn{6}{|l|}{ Comorbilidad (Diabetes) } \\
\hline Presente & $1(20,0)$ & $51(67,1)$ & 5 & $(100)$ & 0,84 \\
\hline Ausente & $25(23,8)$ & $26(86,7)$ & 105 & $(100)$ & \\
\hline \multicolumn{6}{|l|}{ Tipo de tuberculosis } \\
\hline Sensible & $14(23,0)$ & $47(77,0)$ & 61 & $(100)$ & 0,00 \\
\hline MDR & $23(46,9)$ & $26(53,1)$ & 49 & $(100)$ & \\
\hline \multicolumn{6}{|l|}{ Estatus del tratamiento } \\
\hline Antes tratado & $8(66,7)$ & $4(33,3)$ & 12 & $(100)$ & 0,00 \\
\hline Paciente nuevo & $23(23,5)$ & $75(76,5)$ & 98 & $(100)$ & \\
\hline
\end{tabular}




\section{DISCUSIÓN}

En el mundo se considera que 322 millones de personas viven con depresión y no existen muchos datos sobre la relación entre tuberculosis y depresión. Los estudios dan cuenta de que la prevalencia de depresión oscila entre el $17 \%$ y el $54 \%{ }^{7-32}$. En este estudio, el porcentaje de depresión en los pacientes con tuberculosis fue del $54,5 \%$, superior a lo informado por Ugarte et al. ${ }^{22}$ para el Perú y lo reportado en otros estudios realizados en $\operatorname{India}^{28}(39,5 \%)$, Grecia ${ }^{29}$ $(49,2 \%)$, Etiopía ${ }^{16}(43,3 \%)$, Nigeria ${ }^{15}$ $(45,5 \%)$, dos estudios en Pakistán ${ }^{20,30}$ $(69,6 \%, 46,5 \%)$ y $\operatorname{Tanzania}^{31}(46,9 \%)$.

En el Perú, las enfermedades mentales están creciendo y son causa de sufrimiento y de carga para el sistema de salud, puesto que la atención psiquiátrica muchas veces no se encuentra disponible en algunas zonas apartadas del país. Así mismo, son causa de discriminación y en algunos casos de discapacidad, lo que contribuye al empobrecimiento de las personas que padecen la enfermedad ${ }^{32}$.

Koyanagi et al..$^{33}$ realizó un estudio donde evaluó la Encuesta Mundial de Salud Mundial de 48 países de ingresos medios ybajos, y encontró quelos pacientes que presentan tuberculosis tienen 4 veces más riesgo de desarrollar episodios de depresión. De la misma manera, Oh et al. ${ }^{7}$, en un estudio de cohorte realizado en Corea del Sur durante 10 años, encontró que el riesgo de desarrollar tuberculosis cuando se está deprimido aumentaba 3 veces más, comparado con las personas sin depresión.

El presente estudio encontró asociación entre la depresión y el género, identificando mayor prevalencia entre las mujeres, lo cual concuerda con la literatura, donde la depresión es más preponderante en dicha población por factores biológicos, genéticos y hormonales. Así mismo, las mujeres se encuentran más expuestas y son más vulnerables a estresores psicosociales ${ }^{12,14,34}$.

El estudio evidenció también más frecuencia de depresión entre los pacientes que recibían un salario igual o menor al salario mínimo, situación que permite interpretar la incidencia del efecto de la situación financiera en el desarrollo de la depresión entre los pacientes, coincidiendo con estudios como el de Sulerhi et al. ${ }^{35}$ y Salodia et al. ${ }^{36}$.

El estudio muestra asociación entre los niveles de depresión, el tipo de tuberculosis y el estatus del tratamiento. Los pacientes con TBMDR, y tratados anteriormente, presentaron mayor prevalencia de depresión. Esta situación puede estar relacionada, considerando que medicamentos como la cicloserina y etionamida -que hacen parte de los esquemas para el tratamiento de la multidrogorresistencia- contribuyen al desarrollo de la depresión en el paciente ${ }^{37}$. Sin embargo, por ser este estudio de tipo transversal, no se llegó a establecer una relación causal entre el fármaco y el desarrollo de la depresión.

\section{CONCLUSIONES}

Se encontró un porcentaje de depresión alto en comparación con otros estudios tanto nacionales como internacionales, lo que implica que el personal de salud -en la evaluación de los pacientes-deba mantener un alto índice de sospecha de depresión, sobre todo en aquellos que presentan factores de riesgo como el género, baja escolaridad y nivel socioeconómico bajo, 
los cuales son especialmente propensos a esta enfermedad. Por tanto, se recomienda que los programas de tuberculosis realicen evaluaciones acerca de la salud mental de los pacientes en tratamiento, de tal manera que se brinden intervenciones integrales y multitransdisciplinarias para un manejo adecuado de quienes lo necesiten. Así mismo, tanto el personal de salud como la familia del paciente deben ser capacitados para la detección precoz de los síntomas de la depresión, de tal manera que en caso el paciente presente un síntoma de alarma pueda ser derivado para su atención especializada.

\section{REFERENCIAS BIBLIOGRÁFICAS}

1. World Health Organization Global tuberculosis report, 2019. Disponible en: https://www.who.int/tb/publications/global_report/en/.

2. Laxminarayan R, Sridhar D, Blaser M, Wang M, Woolhouse M. Achieving global targets for antimicrobial resistance. Science. 2016; 353: 874-875.

3. Murray CJL, Ortblad KF, Guinovart C, Lim SS, Wolock TM, Roberts DA, et al. Global, regional, and national incidence and mortality for HIV, tuberculosis, and malaria during 1990-2013: A systematic analysis for the Global Burden of Disease Study 2013. Lancet. 2014 Jan 1;384(9947):1005-70.

4. Tiemersma EW, Van der Werf MJ, Borgdorff MW et al. Natural history of tuberculosis: duration and fatality of untreated pulmonary tuberculosis in HIV negative patients: a systematic review. PLoS one 2011;6:e17601. doi:10.1371/journal.pone.0017601.

5. Sharma SK, Mohanan S, Sharma A. Relevance of latent $T B$ infection in areas of high TB prevalence. Chest 2012;142:761-73.doi:10.1378/ chest.12-0142.
6. Rivera $\mathrm{O}$, Benites S, Mendigure J, Bonilla CA. Abandono del tratamiento en tuberculosis multirresistente: factores asociados en una región con alta carga de la enfermedad en Perú. Biomédica. 2019 Aug 1;39(Supl. 2):44-57.

7. Oh K, Choi H, Kim E, Kim H, Cho S. Depresión y riesgo de tuberculosis: un estudio de cohorte basado en la población a nivel nacional. Int J Tuberculosis Lung Dis . 2017; 21 (7): 804-809. doi: 10.5588 / ijtld.17.0038.

8. Koyanagi A, Vancampfort D, Carvalho AF y col. Depresión comórbida con tuberculosis y su impacto en el estado de salud: análisis transversal de datos basados en la comunidad de 48 países de bajos y medianos ingresos. BMC Med . 2017; 15 (1): 209. doi: 10.1186 / s12916-017-0975-5.

9. Eddy M. Depression: The disorder and the burden. Indian J Psychol Med . 2010; 32 (1): 1. doi: 10.4103 / 0253 7176.70510 .

10. Vigo D, Thornicroft G, Atun R. Estimating the true global burden of mental illness. Lancet Psychiatry . 2016; 3 (2): 171-178. doi: 10.1016 / S22150366 (15) 00505-2.

11. World Health Organization. Health Topics on Depression. Available from: http://www.who.int/topics/depression/ en/. Consulta: 19 octubre del 2019.

12. Ambaw F, Mayston R, Hanlon C, Medhin G, Alem A. Untreated depression and tuberculosis treatment outcomes, quality of life and disability, Ethiopia. Bull World Health Organ. 2018;96(4):243. doi:10.2471/ BLT.17.206417.

13. Peltzer K, Naidoo P, Matseke G, Louw J, Mchunu G, Tutshana B. Prevalence of psychological distress and associated factors in tuberculosis patients in public primary care clinics 
in South Africa. BMC Psychiatry. 2012;12(1):89. doi:10.1186/1471244X-12-86.

14. Issa BA, Yussuf AD, Kuranga SI. Depression comorbidity among patients with tuberculosis in a university teaching hospital outpatient clinic in Nigeria. Ment Health Fam Med. 2009;6(3):133.

15. Ige OM, Lasebikan VO. Prevalence of depression in tuberculosis patients in comparison with non-tuberculosis family contacts visiting the DOTS clinic in a Nigerian tertiary care hospital and its correlation with disease pattern. Ment Health Fam Med. $2011 ; 8(4): 235$.

16. Duko B, Gebeyehu A, Ayano G. Prevalence and correlates of depression and anxiety among patients with tuberculosis at WolaitaSodo University Hospital and Sodo Health Center, WolaitaSodo, South Ethiopia, Cross sectional study. BMC Psychiatry. 2015;15(1):214. doi:10.1186/ s12888-015-0598-3.

17. Gelaye B, Williams MA, Lemma S et al. Validity of the patient health questionnaire-9 for depression screening and diagnosis in East Africa. Psychiatry Res. 2013;210(2):653-661. doi: 10.1016/j.psychres.2013.07.015.

18. Bitew H, Andargie G, Tadesse A, Belete A, Fekadu W, Mekonen T. Suicidal ideation, attempt, and determining factors among HIV/AIDS patients, Ethiopia. Depress Res Treat. 2016;2016.

19. Molla A, Mengesha A, Derjaew H, Kerebih $\mathrm{H}$. Suicidal ideation, attempt, and associated factors among patients with tuberculosis in Ethiopia: a cross-sectional study. Psychiatry J. 2019;2019.

20. Javaid A, Mehreen S, Khan MA, Ashiq N, Ihtesham M. Depression and its associated factors with multidrug-resistant tuberculosis at baseline. J Depress Anxiety. 2017;6:253.

21. Walker I, Khan AM, Khan AM et al. Depression among multidrug-resistant tuberculosis patients in Punjab, Pakistan: a large cross-sectional study. Int J Tuberc Lung Dis. 2018;22:773-778.

22. Ugarte-Gil C, Ruiz P, Zamudio C, Canaza L, Otero L, Kruger H et al. Association of Major Depressive Episode with Negative Outcomes of Tuberculosis Treatment. PLoS One. 2013 Jul 29;8(7).

23. Caballero P, Yagui $M$, Espinoza $M$, Castilla T, Granados A, Velásquez A et al. Prioridades regionales y nacionales de investigación en salud, Perú 2010-2014: un proceso con enfoque participativo y descentralista. Rev Peru Med Exp Salud Publica. 2010 Sep;27(3):398-411.

24. Kocalevent RD, Hinz A, Brähler E. Standardization of the depression screener Patient Health Questionnaire (PHQ9) in the general population. Gen Hosp Psychiatry. 2013;35:551-5. http://dx.doi.org/10.1016/j.genhosppsych.2013.04.006.

25. Kroenke K, Spitzer RL, Williams JB. The PHQ-9: Validity of a brief depression severity measure. Gen Intern Med. 2001;16:606-13. http://dx.doi. org/10.1046/j.1525-1497.2001. 016009606.x.

26. Williams N. PHQ-9. Occup Med (Lond). 2014;64:139-40. http://dx. doi.org/10.1093/occmed/kqt154.

27. Sung SC, Low CC, Fung DS. Screening for major and minor depression in a multiethnic sample of Asian primary care patients: A comparison of the nine-item Patient Health Questionnaire (PHQ-9) and the 16-item Quick Inventory of Depressive Symptomato- 
logy - Self-Report (QIDS-SR16). Asia Pac Psychiatry. 2013;5:249-58.http:// dx.doi.org/10.1111/appy.12101.

28. Balaji AL, Abhishekh HA, Kumar NC, Mehta RM. Depression in patients with pulmonary tuberculosis in a tertiary care general hospital. Asian J Psychiatr. 2013;6(3):251-252. doi:10.1016/j.ajp.2012.12.017.

29. Moussas G, Tselebis A, Karkanias A et al. A comparative study of anxiety and depression in patients with bronchial asthma, chronic obstructive pulmonary disease and tuberculosis in a general hospital of chest diseases. Ann Gen Psychiatry. 2008;7(1):7. doi:10.1186/1744-859X-7-7.

30. Husain MO, Dearman SP, Chaudhry IB, Rizvi N, Waheed W. The relationship between anxiety, depression and illness perception in tberculosis patients in Pakistan. Clin Pract Epidemiol Mental Health. 2008;4(1):4. doi: 10.1186/1745-0179-4-4.

31. Buberwa G. Prevalence of Depression among Tuberculosis Patients Attending Clinics in Temeke Municipal, Dar Es Salaam. Tanzania: Muhimbili University of Health and Allied Sciences; 2013.

32. Berndt ER, Koran LM, Finkelstein SN, Gelenberg AJ, Kornstein SG, Miller IM, et al. Lost human capital from early-onset chronic depression. Am J Psychiatry 2000; 157(6): 940-47.

33. Koyanagi A, Vancampfort D, Carvalho AF, DeVylder JE, Haro JM, Pizzol D, Veronese N, Stubbs B. Depression comorbid with tuberculosis and its impact on health status: cross-sectional analysis of community-based data from 48 low- and middle-income countries. BMC medicine. 2017; 15(1):209.

34. Harkness KL, Alavi N, Monroe SM, Slavich GM, Gotlib IH, Bagby RM. Gender differences in life events prior to onset of major depressive disorder: The moderating effect of age. J Abnorm Psychol 2012; 119(4): 791-803.

35. Sulehri MA, Dogar IA, Mehdi HS, Azam M, Niaz O, Javed MS et al. Prevalence of depression among tuberculosis patients. APMC 2010; 4:133-7.

36. Salodia UP, Sethi S, Khokhar A. Depression among tuberculosis patients attending a DOTS centre in a rural area of Delhi: A cross-sectional study. Indian J Public Health. 2019 Jan 1;63(1):39-43.

37. Vega P, Sweetland A, Acha J, Castillo H, Guerra D, Smith Fawzi MC et al. Psychiatric issues in the management of patients with multidrug-resistant tuberculosis. Int J Tuberc Lung Dis 2004;8:749-59. 